\title{
PERTURBATION OF ZEROS IN THE PRESENCE OF SYMMETRIES
}

\author{
E. N. DANCER
}

(Received 14 May 1982)

Communicated by R. O. Vyborny

\begin{abstract}
We study the existence of zeros of a perturbed nonlinear operator near a zero of the unperturbed operator in the case where both operators are invariant under a symmetry group. To do this, we first correct some work of Rubinsztein on the $G$-homotopy groups of spheres.
\end{abstract}

1980 Mathematics subject classification (Amer. Math. Soc.): 58 E 07, 47 H 15, 57 S 99.

The problem we wish to study is the following. Assume that $G$ is a compact Lie group, $\left\{T_{g}\right\}_{g \in G}$ is a linear action of $G$ on a finite-dimensional linear space $E$, and $F: E \times R \rightarrow E$ is continuous and $G$-invariant (that is, $F\left(T_{g} x, t\right)=T_{g} F(x, t)$ for $g \in G, x \in E, t \in R$ ). Finally assume that $k \in E$ such that $F(k, 0)=0$ and that $F(x, 0) \neq 0$ in some deleted neighbourhood of $M$, where $M$ denotes the orbit $G(k) \equiv\left\{T_{g} k: g \in G\right\}$. (Note that, by the symmetries, $F(, 0)$ vanishes on $M$.) We want to find necessary and sufficient conditions on $F(, 0)$ for the equation $F(x, t)=0$ to have solutions near $M$ for all $t$ near 0 . Note that, in the case where $G=\{e\}$, it is a well-known result that this holds if and only if $\operatorname{index}_{E}(F, k) \neq 0$. (The index is defined in Lloyd [18].) Surprisingly, we show that the conditions on $F(, 0)$ are different if we assume that $F$ is $C^{1}$ (but still require that $F(x, t)=0$ has solutions near $M$ for all $t$ near 0 ).

We should explain what is already known. In [6], we obtained a $G$-invariant implicit function theorem, in other words, an extension of the implicit function theorem to the invariant case. Our main result here gives weaker conditions for the existence (but not the uniqueness) of orbits of zeros of $F(, t)$ near $M$. Thus our main result bears the same relationship to the $G$-invariant implicit function

(C) 1984 Australian Mathematical Society $0263-6115 / 84 \$ A 2.00+0.00$ 
theorem as does the classical degree theory result mentioned at the end of previous paragraph to the ordinary implicit function theorem. On the other hand, Golubitsky and Schaefer [13] have some related work on the zeros of perturbations in the invariant case when $M=\{0\}$ (that is, when $k=0$ ). Their results, which apply to smooth maps, give in principle a nearly complete analysis of the number of zeros of all smooth perturbations of $F(, 0)$ provided $F(, 0)$ has finite $G$-codimension. (We do not explain this here because we shall not need it.) They do this by looking at $G$-universal unfoldings. Thus their results are much stronger than ours when they can be applied. Note that the assumption that $M=\{0\}$ severely restricts the use of their results. The case where $M=\{0\}$ seems to be significantly simpler than the general case. Moreover, our work, which shows the occurrence of normalizer conditions, should help in understanding what their method involves for complicated group actions. In addition, our results are also applicable if $F(, 0)$ does not have finite $G$-codimension. Moreover, even when their results are applicable, the calculations with the universal unfolding are difficult and tedious except in very simple cases especially if the $G$-codimension is large. Thus it may happen that our results can be used while theirs cannot, simply because one cannot do the calculations in their methods. Note that, to verify the assumptions of our theorem, we only need to calculate a number of normalizer groups and a number of degrees of mappings. These are usually reasonably easy to calculate. Thus our results and theirs seem complementary.

It might be asked why we are interested in our problem at all. This sort of problem occurs in bifurcation theory when an invertibility condition fails. See [5], [6] or [13].

Our proofs depend essentially upon some work of Rubinsztein [21] on the equivariant homotopy of spheres. Unfortunately, as pointed out in Komiya [17], the main theorem in [21] contains an error. We give a corrected statement of his main result and explain briefly how the proof has to be altered. We also give an alternative formulation of his main theorem which seems convenient for calculations. Note that it is also of interest to correct Rubinsztein's work because we used it in [8] to prove a result relating degrees in the symmetric case.

In Section 1, we discuss Rubinsztein's error and its correction and also some variants of his work. We defer any discussion of the proofs of these results to the appendix. There we sketch the proofs. In Section 2, we obtain our main results. In Section 3, we obtain some partial results for the case where $F$ is $C^{1}$ or $F$ is a gradient mapping. Finally, in Section 4, we discuss some special cases and examples. We avoid most of the technicalities of the general case in Section 4.

Because of the rather technical nature of the paper, I should indicate how the sections depend upon each other. To read most of Section 4, one needs to first read the introduction and the first two paragraphs of Section 2. (If the reader 
does this, he should ignore bracketed comments in Section 4. These consider generalizations or explain how the results follow from those of Section 2.) In addition, Section 2 can be read without reading the very technical Section 1 provided that one ignores the proof that the two statements of Theorem 4 are equivalent.

It is assumed that the reader has a knowledge of the basic properties of transformation groups as in Brc don [3, Chapter 1].

\section{On Rubinsztein's results}

In this section, we discuss some results of Rubinsztein which are basic to our work. We point out an error in his work and state a corrected version of his main theorem. We also discuss some minor extensions of his work and also a variant of his main theorem which seems more convenient for calculations. We merely state the results here and defer a brief discussion of the proofs to the appendix. We do this because these proofs are quite separate from the main interest of this paper.

We assume that $S(V)$ is the unit sphere in a finite-dimensional linear space $V$ on which the compact Lie group $G$ acts linearly and unitarily by the action $\left\{T_{g}\right.$ : $g \in G\}$. Rubinsztein establishes a 1-1 correspondence between $[S(V), S(V)]_{G}$, the set of $G$-homotopy classes of $G$-invariant maps of $S(V)$ into itself and a subset of $Z^{k}$ for some $k$. We will be more precise in a moment. Note that, for simplicity (and also because we will not need the more general results), we are assuming that $W=\{0\}$ (with the notation of [21]).

Firstly, we explain briefly his error. He assumes that, if $K \subseteq H \subseteq G$ where $K$ and $H$ are closed subgroups of $G$, then $(G / H)^{K} \equiv\{g H \in G / H: k g H=g H$ for all $k$ in $K$ \} is a manifold. Now general theory (see Bredon [3, Corollary 6.2.5]) ensures that each component of $(G / H)^{K}$ is a manifold but one can construct simple examples where the components have different dimensions. (Note that Bredon's result is stated incorrectly.) This error was pointed out in [17].

Let us now state a corrected version of Rubinsztein's reslt. We first introduce quickly his basic notation. If $H$ is a closed subgroup of $G$ and $A \subseteq V$, let $A^{H}$ be the set of points in $A$ fixed by $H$, let $X^{H}=\left\{x \in S(V): G_{x}=H\right\}$ where $G_{x}$ denotes the isotropy group of $x$ and let $X_{(H)}$ be $\left\{T_{g} x: g \in G, x \in X^{H}\right\}$.

Define $\Re$ to be the set of isotropy groups $H$ (up to conjugacy) for the action of $G$ on $S(V)$ with the additional property that $N_{G}(H) / H$ is finite, where $N_{G}(H)$ denotes the normalizer of $H$ in $G$. Finally, let $\pi$ denote the natural map of $V$ into the quotient space $V / G$.

We assume a knowledge of the rest of his notation in Section 7 of [21]. (There seems little point in repeating all his definitions. We will not need them in later 
sections.) The one change that has to be made is that the definition of $\kappa_{\tilde{H}}$ has to be modified. We define $\kappa_{\tilde{H}}\left(1_{((H), \alpha)}\right)=\Sigma(-1)^{t_{i}} \chi\left(\alpha_{i}\right)$, where the summation is over the components $\alpha_{i}$ of $(G / H)^{H}$ and $t_{i}=\operatorname{dim} \alpha_{i}$. With this change his main theorem is true. Thus we have the following result.

THEOREM 1. There is a 1-1 correspondence $\Psi$ between $[S(V), S(V)]_{G}$ and $A(V,\{0\})$ such that, if $H$ is a closed subgroup of $G$ and if $f$ is a representative of $a$ homotopy class $[f]$ in $[S(V), S(V)]_{G}$, then $\operatorname{deg}_{S(V)^{H}}\left(\left.f\right|_{S(V)^{H}}\right)=\kappa_{H} \Psi[f]$.

Note that, if $S(V)^{H}$ is empty, we take the degree to be 1. We will explain how the original proof can be modified in the appendix.

The correspondence in Theorem 1 does not give a very convenient method for deciding whether maps are $G$-homotopic. We now present a more explicit criteria. First we need some notation. If $H \in \mathfrak{R}$ consider $W_{H}=\left\{x \in S(V)^{H}: G_{x} \neq H\right\}$. This is a closed proper subset of $S(V)^{H}$. Suppose $T$ is a component of $\pi X_{(H)}$. Let $\tilde{T}=\pi^{-1} T \cap S(V)^{H}$ and choose $p \in \tilde{T}$. Note that $\tilde{T}$ is open in $S(V)^{H}$. If $f$ : $S(V) \rightarrow S(V)$ is $G$-invariant, one easily sees that $f$ maps $S(V)^{H}$ into itself and $W_{H}$ into itself. Since $\partial\left(X^{H}\right) \subseteq W_{H}$ and thus $f(x) \neq p$ for $x \in \partial\left(X^{H}\right)$, it follows that $\operatorname{deg}_{S(V)^{H}}(f, p, \tilde{T})$ is defined and is independent of $p$ for $p$ in a component of $\tilde{T}$ (by homotopy invariance). Note that $N(H) / H$ acts freely on $S(V)^{H} \backslash W_{H}$. Thus if $g \in N(H) / H$ and $T^{1}$ is a component of $S(V)^{H} \backslash W^{H}$, then $\tilde{T}^{1}=T_{g} T^{1}$ is also a component of $S(V)^{H} \backslash W^{H}$ (possibly $T^{1}$ again). By the commutativity theorem for the degree and the $G$-invariance of $f, \operatorname{deg}_{S(V)^{H}}\left(f, p, T^{1}\right)=\operatorname{deg}_{S(V)^{H}}\left(f, T_{g} p, \tilde{T}^{1}\right)$. By summing over the components of $\tilde{T}$, we find that $\operatorname{deg}_{S(V)^{H}}(f, p, \tilde{T})=$ $\operatorname{deg}_{S(V)^{H}}\left(f, T_{g} p, \tilde{T}\right)$. Thus $\operatorname{deg}_{S(V)^{H}}(f, p, \tilde{T})$ is constant for $p$ in $\tilde{T}$. Note that since $f(x) \neq p$ on $\partial \tilde{T}$, we easily see that these degrees are $G$-homotopy invariants of $f$. Moreover, if $\pi X_{(H)}$ is connected, then $\tilde{T}$ has closure $S(V)^{H}$. Thus, since $f(x) \neq p$ on $\partial \tilde{T}, \operatorname{deg}_{S(V)^{H}}(f, p, \tilde{T})=\operatorname{deg}_{S(V)^{H}}\left(f, p, S(V)^{H}\right)=\operatorname{deg}_{S(V)^{H}}(f)$. This degree is often easier to calculate. (It equals index $V_{V^{H}}\left(\left.F\right|_{V^{H}}, 0\right)$ if $f(x)=$ $\|F(x)\|^{-1} F(x)$ where $F: V \rightarrow V$ is continuous, $G$-invariant and $F(x) \neq 0$ for $x \neq 0$.)

Let $Z$ denote the set of pairs $(H, \tilde{T})$ such that $H \in \mathfrak{N}$ and $\tilde{T}=\pi^{-1}(T) \cap S(V)^{H}$, where $T$ is a component of $\pi X_{(H)}$.

Theorem 2. Suppose $f_{1}, f_{2}: S(V) \rightarrow S(V)$ are G-invariant. Then they are $G$-invariantly homotopic if and only if $\operatorname{deg}_{S(V)^{H}}\left(f_{1}, p, \tilde{T}\right)=\operatorname{deg}_{S(V)^{H}}\left(f_{2}, p, \tilde{T}\right)$ for each $(H, \tilde{T}) \in Z$. (Here $p \in \tilde{T}$.)

The advantage of this variant of Theorem 1 is that degrees are often relatively easy to calculate. For example, the degree is often easy to calculate if $f_{1}$ is smooth 
and $p$ is a regular value of $f_{1} \mid \tilde{T}$. Note that our comments above prove the necessity of the conditions. Once again, we prove Theorem 2 in the appendix.

Finally, assume that $Y$ is a subset of $S(V)$ with the property that if $x \in Y$ and $G_{y}$ contains a conjugate of $G_{x}$, then $y \in Y$. Standard theory implies that $Y$ is $G$-invariant and closed and is a union of $X_{(H)}$ 's. Let $Z_{Y}=\left\{(H, \tilde{T}) \in Z: G_{x}=H\right.$ for some $x$ in $Y$ \}. Thus $Z_{Y}$ is the part of $Z$ corresponding to points in $Y$.

THEOREM 3. Suppose $f_{1}, f_{2}: Y \rightarrow S(V)$ are continuous and $G$-invariant. Then they are G-invariantly homotopic if and only if $\operatorname{deg}_{S(V)^{H}}\left(f_{1}, p, \tilde{T}\right)=\operatorname{deg}_{S(V)^{H}}\left(f_{2}, p, \tilde{T}\right)$ for every $(H, \tilde{T}) \in Z_{Y}$.

This is a slight strengthening of Theorem 2. Once again it is proved in the appendix. We need Theorem 3 in Section 2.

\section{The main result}

We assume that $\left\{T_{g}\right\}_{g \in G}$ is a linear action of a compact Lie group $G$ on a finite-dimensional linear space $E, \mathscr{F}: E \rightarrow E$ is continuous and $G$-invariant, $\mathscr{F}(k)=0$ and there is a neighbourhood $\mathcal{T}$ of $M \equiv G(k)$ such that $\mathscr{F}(x) \neq 0$ for $x \in \mathcal{G} \backslash M$. Let $K=G_{k}$, where $G_{k}=\left\{g \in G: T_{g} k=k\right\}$. As usual, we may assume without loss of generality that each $T_{g}$ is unitary (see [3, Theorem 6.2.1]). By the tubular neighbourhood theorem (see [3, Theorem 6.2.2]), we may choose $\mathcal{T}$ to be a $G$-invariant neighbourhood of $M$ such that $\mathscr{T}$ intersects $N_{k}(M) \equiv T_{k}(M)^{\perp}$ in a ball $B_{r}(k)$ in $N_{k}(M)$. Let $P_{k}$ be the orthogonal projection onto $N_{k}(M)$. It is easy to check that $P_{k}$ is $K$-invariant. Let $K^{i}, i=1, \ldots, m$, denote the isotropy groups (up to conjugacy in $K$ ) for the action of $K$ on $N_{k}(M)$, where $K^{1}=K$. (It is easy to check that $N_{k}(M)$ is $K$-invariant.) As usual, we may order the $K^{i}$ such that $K^{i}$ is not conjugate (in $K$ ) to a subgroup of $K^{j}$ if $i \leqslant j$. If $A \subseteq E$ and $H$ is a subgroup of $G$, let $A^{H}=\left\{x \in A: T_{g} x=x\right.$ for all $\left.g \in H\right\}$. Let $N_{i}=T_{k}(M)^{K^{i}}$ and $\tilde{N}_{i}=$ $N_{k}(M)^{K^{i}}$. We will discuss the $N_{i}$ later in this section.

We say that $k$ is $G$-stable for $\mathcal{F}$ if there is a $\delta>0$ such that every continuous $G$-invariant map $\tilde{F}$ such that $\|\mathscr{F}(x)-\tilde{F}(x)\| \leqslant \delta$ on $T$ has a zero in $\tilde{T}$. It is easy to see that this definition is independent of the choice of $\mathcal{T}$ (in particular, independent of the $r$ in the definition of $\mathscr{T}$ ). In the main result of this section, we obtain necessary and sufficient conditions for $F$ to be $G$-stable.

Since $\left.P_{k} \mathscr{F}\right|_{N_{k}(M)}$ is $K$-invariant, it follows easily that $P_{k} \mathscr{F}$ maps $\tilde{N}_{i}$ into itself. Suppose that $N_{i}=\{0\}$ and $x \in \tilde{N}_{i} \cap \mathcal{G}$ such that $P_{k} \mathscr{F}(x)=0$. Because $\mathscr{F}$ is $K$-invariant, we easily see that $\mathscr{F}(x)$ is fixed by $K^{i}$. Since $P_{k}$ is $K$-invariant, we also see that $\left(I-P_{k}\right) \mathscr{F}(x)$ is fixed by $K^{i}$. Thus it is in $N_{i}$ and hence is zero. Thus 
$\mathscr{F}(x)=0$. Hence, by our assumptions on $\mathscr{F}, x \in M$. Thus we see that, if $N_{i}=\{0\}$, then $P_{k} \mathscr{F}(x) \neq 0$ on $\left(\tilde{N}_{i} \cap B_{r}(k)\right) \backslash\{k\}$. Let $N^{i}$ denote the set of points on the sphere $S_{r}(k)$ in $\Re_{k}(M)$ with centre $k$ which have isotropy group conjugate to $K^{i}$ and let $\bar{N}^{i}$ be the closure of $N^{i}$. It is well known that, for each point $x$ in $\bar{N}^{i}$, the isotropy group $K_{x}$ has some conjugate of $K^{i}$ as a subgroup. Now it is easy to show that, if $K^{i}$ is conjugate (in $K$ ) to a subgroup of $K^{j}$ and $N_{i}=\{0\}$, then $N_{j}=\{0\}$. It follows as above that $P_{k} \mathscr{F}(x) \neq 0$ on $S_{r}(k) \cap \bar{N}^{i}$. Let $f_{i}: \bar{N}^{i} \rightarrow S_{r}(k)$ be defined by $f_{i}(x)=k+r\left\|P_{k} \mathscr{F}(x)\right\|^{-1} P_{k} \mathscr{F}(x)$. It is easy to check that $f_{i}$ is $K$-invariant. This is the type of map we considered in Theorem 3 (if we use a change of origin by $k$ and a change of scalar by $r$ ). The following theorem is the main result of this paper.

THEOREM 4. $\mathscr{F}$ is $G$-stable if and only if there is an $i$ in $\{1, \ldots, m\}$ such that $N_{i}=\{0\}$ and $f_{i}$ is not $K$-invariantly homotopic to a constant map. Equivalently, $\mathscr{F}$ is $G$-stable if and only if (I) there is an $i$ and a component $T$ of $\tilde{\pi}\left(N^{i}\right)$ such that (a) $N_{i}=\{0\}$, (b) $\operatorname{dim} N_{K}\left(K^{i}\right)=\operatorname{dim} K^{i}$, where $N_{K}\left(K^{i}\right)$ denotes the normalizer of $K^{i}$ in $K$, (c) $\operatorname{dim} \tilde{N}_{i}>1$ and (d) $\operatorname{deg}_{S_{r}(k)} \kappa_{i}\left(f_{i}, p, \tilde{\pi}^{-1}(T) \cap \tilde{N}_{i}\right) \neq 0$ where $p \in$ $\tilde{\pi}^{-1}(T) \cap \tilde{N}_{i}$ or (II) there is an $i$ such that $N_{i}=\{0\}, \operatorname{dim} \tilde{N}_{i}=1$ and $f_{i} \mid \tilde{N}_{i} \cap N^{i}$ is not a constant map. Here $\tilde{\pi}$ is the natural map of $S_{r}(k)$ into the orbit space $S_{r}(k) / K$.

Remark. It is possible to replace (a) and (b) by the single condition that $\operatorname{dim} N_{G}\left(K^{i}\right)=\operatorname{dim} K^{i}$. The proof of this uses our later discussion of $N_{i}$ and also requires the consideration of the natural map of $G / K^{i}$ to $G / K$. It is unclear which formulation is more convenient in applications. Note that Theorem 4 gives conditions which are reasonable to check in applications.

Proof. The second statement follows from the first and Theorem 3 of Section 1 (applied to the action of $K$ on $\bar{N}^{i} \subseteq S_{r}(k)$ ). Thus we need only consider the first statement.

We first prove that $\mathscr{F}$ is $G$-stable if there is an $i$ such that $N_{i}=\{0\}$ and $f_{i}$ is not $K$-invariantly homotopic to a constant. Since $S_{r}(k)$ is a $K$-invariant strong deformation retract of $\Re_{k}(M) \backslash\{k\}$ (by the obvious retraction), it follows that the map $x \rightarrow P_{k} \mathscr{F}(x)$ is not $K$-invariantly null homotopic as a map of $\bar{N}^{i}$ into $\Re_{k}(M) \backslash\{0\}$. First note that, since $\bar{N}^{i}$ is compact, there is a $\mu>0$ such that $\left\|P_{k} \mathscr{F}(x)\right\| \geqslant \mu$ on $\bar{N}^{i}$. It follows easily that if $\tilde{F}: \mathscr{T} \rightarrow E$ is continuous and $G$-invariant and if $\|\mathscr{F}(x)-\tilde{F}(x)\| \leqslant \frac{1}{2} \mu$ on $\mathscr{T}$, then $P_{k} \mathscr{F}$ and $\left.P_{k} \tilde{F}\right|_{\overline{N^{i}}}$ are $K$-invariantly homotopic as maps of $\bar{N}^{i}$ into $\mathscr{T}_{k}(M) \backslash\{0\}$. (We simply use the map $(x, t) \rightarrow t P_{k} \mathscr{F}(x)+(1-t) P_{k} \tilde{F}(x)$.) Thus $\left.P_{k} \tilde{F}\right|_{N^{i}}$ is not $K$-invariantly homotopic to a constant map. It follows that there exists $\tilde{x} \in C \bar{N}^{i} \equiv\{x: x=k+t y$, $\left.0 \leqslant t \leqslant 1, y \in \bar{N}^{i}\right\}$ such that $P_{k} \tilde{F}(\tilde{x})=0$. [If $P_{k} \tilde{F}(x) \neq 0$ on $C \bar{N}^{i}$, the map 
$(x, t) \rightarrow P_{k} \tilde{F}(t x+(1-t) k)$ (as a map of $\left.\bar{N}^{i} \rightarrow \Re_{k}(M) \backslash\{0\}\right)$ would be a $K$-invariant homotopy to a constant map.] Now $\tilde{x}=T_{k} y$ where $k \in K$ and $y$ has isotropy group a subgroup containing $K^{i}$. (Remember that any $x$ in $\bar{N}^{i}$ has an isotropy group which contains a conjugate of $K^{i}$.) By $K$-invariance, $P_{k} \tilde{F}(y)=0$. Since $y$ is fixed by $K^{i},\left(I-P_{k}\right) \tilde{F}(y)$ is fixed by $K^{i}$ and is contained in $T_{k}(M)$. Hence, since $N_{i}=\{0\},\left(I-P_{k}\right) \tilde{F}(y)=0$. Thus $\tilde{F}(y)=0$. Hence $\mathscr{F}$ is $G$-stable. (Take $\delta=\frac{1}{2} \mu$.)

To prove the converse, we will show that $\mathscr{F}$ is not $G$-stable if, for each $i$ such that $N_{i}=\{0\}, f_{i}$ is $K$-invariantly homotopic to a constant map. We need a lemma.

LemMa 1. If $f: B_{r}(k) \rightarrow \mathfrak{R}_{k}(M)$ is a continuous $K$-invariant map such that $f(x) \neq 0$ on $S_{r}(k)$ and $\delta>0$, then there is a K-invariant map $\tilde{f}: B_{r}(k) \rightarrow \Re_{k}(M)$ such that (i) $\|\tilde{f}(x)-f(x)\| \leqslant \delta$ on $B_{r}(k)$, (ii) $\tilde{f}$ vanishes on only a finite number of (K-) orbits in $B_{r}(k)$ and (iii) $\tilde{f}(x)=f(x)$ on $S_{r}(k)$.

Proof. By applying Theorem 6.4.2 in Bredon [3] with $M=$ int $B_{r}(k)$, we see that there is a $K$-invariant map $f_{1}$ such that $f_{1}=f$ on $S_{r}(k),\left\|f_{1}(x)-f(x)\right\| \leqslant \frac{1}{2} \delta$ on $B_{r}(k)$ and $f_{1}$ is smooth in int $B_{r}(k)$. By using the theory of $G$-transversality (see Bierstone [2] and Field [12]) we can approximate $f_{1}$ to within $\frac{1}{2} \delta$ by a $K$-invariant map $\tilde{f}$ such that $\tilde{f}=f$ near $S_{r}(k), \tilde{f}$ is smooth in int $B_{r}(k)$ and $\tilde{f}$ is $K$-transversal to zero. It follows easily that $\tilde{f}$ has the required properties. (The $K$-transversality to zero ensures that $\tilde{f}$ vanishes on only a finite number of orbits.)

Proof of Theorem 4 (continued). We now return to the proof of Theorem 4. If $0<s<r$, we construct a $K$-invariant map $F_{2}: B_{s}(k) \rightarrow E \backslash\{0\}$ such that $\mathscr{F}(x)=F_{2}(x)$ on $S_{s}(k)$ and $\left\|F_{2}(x)\right\| \leqslant q$ on $B_{s}(k)$ where $q=\sup \{\|\mathscr{F}(y)\|: y \in$ $\left.S_{s}(k)\right\}$. This is the key part of the proof. Let $J$ be the set of integers in $\{1, \ldots, m\}$ for which $N_{i}=\{0\}$ and let $A=\left\{x \in S_{s}(k): K_{x}\right.$ is conjugate to $K^{i}$ for some $i$ in $J\}$. By our earlier arguments, $A$ is a closed $K$-invariant subset of $S_{s}(k)$ with the property that, if $x \in A$, if $y \in S_{s}(k)$ and if $K_{y}$ is conjugate (in $K$ ) to a subgroup of $K_{x}$, then $y \in A$. Thus, we can apply Theorem 3 to $P_{k} F \mid A$. Now the obstructions in Theorem 3 to $P_{k} \mathscr{F} l_{A}$ being $K$-invariantly homotopic to a constant (as a map of $A$ into $\left.\Re_{k}(M) \backslash\{0\}\right)$ are precisely all those which occur in the obstructions to each of the $f_{i}, i \in J$, being $K$-invariantly homotopic to a constant. (Here we are not distinguishing between $K$-invariant homotopic maps of $A$ into $S_{s}(k)$ and into $\Re_{k}(M) \backslash\{0\}$ since we can readily change from one to the other.) Thus we find from Theorem 3 and our assumptions on the $f_{i}$ that $P_{k} \mathscr{F}$ is $K$-invariantly homotopic to a constant (as a map of $A$ into $\mathscr{R}_{k}(M) \backslash\{0\}$ ). Let $H$ denote this homotopy, where $H(, 0)=P_{k} \mathscr{F}$. We can now extend $\left.P_{k} \mathscr{F}\right|_{S_{s}(k)}$ to a $K$-invariant map $F_{1}$ of $C A \equiv\{k+t u: 0 \leqslant t \leqslant 1, u \in A\}$ into $\Re_{k}(M) \backslash\{0\}$ by defining 
$F_{1}(k+t u)=H(u, 1-t)$. Define $F_{1}$ to be $\mathcal{F}$ on $S_{s}(k)$. By Theorem 1.2.3 in [3], we can extend $F_{1}$ to a $K$-invariant map of $B_{s}(k)$ into $\Re_{k}(M)$. Since $C A$ is compact and $F_{1}(x) \neq 0$ on $C A$, we can use Lemma 1 (with $\delta$ small) to find a new extension $\tilde{F}_{1}$ of $\left.\mathscr{F}\right|_{S_{s}(k)}$ such that $\tilde{F}_{1}(x) \neq 0$ on $C A$ and $\tilde{F}_{1}(x)$ vanishes on only a finite number of orbits in $B_{s}(k)$. We now perturb $\tilde{F}_{1}$ to construct a $K$-invariant map of $B_{s}(k)$ into $E \backslash\{0\}$. Let $\left\{T_{k} x_{i}: k \in K\right\}$ for $1 \leqslant i \leqslant z$ denote the distinct orbits on which $\tilde{F}_{1}$ vanishes. We can choose the $x_{i}$ such that $K_{x_{i}}=K^{j_{i}}$ for some $j_{i}$ in $\{1, \ldots, m\} \backslash J$. By our construction of $\tilde{F}_{1}$ and the definition of $J, N_{j_{i}} \neq\{0\}$ for $1 \leqslant i \leqslant z$. Suppose $\delta>0$ and choose $v_{i} \in N_{j_{i}}$ with $\left\|v_{i}\right\|=\delta$. We then define a $K$-invariant map $\tilde{F}_{2}$ of $\left\{T_{k} x_{i}: 1 \leqslant i \leqslant z, k \in K\right\}$ into $R\left(I-P_{k}\right)$ by $\tilde{F}_{2}\left(T_{k} x_{i}\right)=$ $T_{k} v_{i}$. (Since $N_{j_{i}}$ is fixed by $K^{j_{i}}$, the map is well-defined and $K$-invariant.) We define $\tilde{F}_{2}$ to be zero on $S_{s}(k)$. By Theorem 1.2.3 in [3], we can extend $\tilde{F}_{2}$ to a $K$-invariant map of $B_{s}(k)$ into $\left\{x \in R\left(I-P_{k}\right):\|x\| \leqslant \delta\right\}$. We now define $F_{2}=\tilde{F}_{1}$ $+\tilde{F}_{2}$. It is easy to see that $F_{2}$ is equal to $\mathscr{F}_{s}$ on $S_{s}(k)$ and $F_{2}(x) \neq 0$ in $B_{s}(k)$. To ensure the last required property of $F_{2}$ we replace $F_{2}$ by $r \circ F_{2}$ where $r$ is the obvious retraction of $E$ onto the ball of radius $q$ in $E$.

We now complete the proof of Theorem 4. Suppose $\varepsilon>0$. Choose $s$ in $(0, r)$ such that $\|\mathscr{F}(x)\| \leqslant \frac{1}{2} \varepsilon$ in $B_{s}(k)$. (Remember that $\|\mathscr{F}(x)\| \rightarrow 0$ as $x \rightarrow k$.) By the result of the previous paragraph, there is a $K$-invariant map $F_{3}: B_{s}(k) \rightarrow E \backslash\{0\}$ such that $\left\|F_{3}(x)\right\| \leqslant \frac{1}{2} \varepsilon$ on $B_{s}(k)$ and $F_{3}(x)=\mathscr{F}(x)$ on $S_{s}(k)$. By the triangle inequality, $\left\|F_{3}(x)-\mathscr{F}(x)\right\| \leqslant \varepsilon$ on $B_{s}(k)$. We extend $F_{3}$ to $B_{r}(k)$ by defining $F_{3}(x)=\mathscr{F}(x)$ if $\|x-k\| \geqslant s$. Finally, we extend $F_{3}$ to the tubular neighbourhood $\mathcal{T}$ by the group invariance. It is then easy to check that $F_{3}$ is continuous and $G$-invariant, $\left\|F_{3}(x)-\mathscr{F}(x)\right\| \leqslant \varepsilon$ on $\mathcal{T}$ and $F_{3}(x) \neq 0$ on $\mathscr{T}$. Since $\varepsilon$ was arbitrary, it follows that $\mathscr{F}$ is not $G$-stable. This completes the proof of Theorem 4.

REMARK. If $\mathscr{F}$ is smooth, a smoothing argument shows that $F_{3}$ can be chosen to be smooth.

The definition of $G$-stability in this section is not the same as in the introduction. The next proposition shows that they are equivalent. We assume that $\mathscr{F}$ satisfies the assumptions at the beginning of the section.

Proposition 1. If $\mathscr{F}$ is not $G$-stable, then there is a continuous G-invariant $F$ : $\mathcal{T} \times[0,1] \rightarrow E$ such that $F(, 0)=\mathscr{F}$ and $F(x, t) \neq 0$ if $t \neq 0$.

Proof. As before, it suffices to construct a $K$-invariant map $F: B_{r}(k) \times[0,1] \rightarrow$ $E$ such that $F(, 0)=\mathscr{F}$ and $F(x, t) \neq 0$ if $t \neq 0$. Since $k$ is fixed by $K$, we can translate axes to assume that $k=0$. Define $F(x, t)=\mathscr{F}(x)$ if $\|x\| \geqslant t$ and $F(x, t)=F_{3}(x)$ if $t \geqslant r$. ( $F_{3}$ was defined in the proof of Theorem 4.) Now it is 
easy to construct a $K$-invariant retraction $\tilde{r}$ of $\left\{(u, t) \in B_{r}(0) \times[0, r]:\|u\| \leqslant\right.$ $t\} \backslash\{(0,0)\}$ onto the subset where $\|u\|=t$ or $t=r$. (One starts with a retraction $r_{1}$ of $\left\{(y, t) \in R^{2}: 0 \leqslant y \leqslant t \leqslant r\right\} \backslash\{(0,0)\}$ onto the subset where $y=t$ or $t=r$ with the property that $r_{1}(0, t)=(0, r)$ for $t \in(0, r]$.) Choose $\alpha:[0, r] \rightarrow(0, \infty)$ to be continuous such that $\alpha(t) \rightarrow 0$ as $t \rightarrow 0$ and $\alpha(t) \geqslant \sup \{\|\mathscr{F}(x)\|:\|x\|=t\}$. Let $R_{q}$ be the obvious retraction of $\mathfrak{X}_{k}(M)$ onto $B_{q}(0)$. Now define $F(x, t)=$ $R_{\alpha(t)} F(\tilde{r}(x, t))$ if $\|x\| \leqslant t \leqslant r$ and $(x, t) \neq(0,0)$. Finally, define $F(0,0)=0$. By our choice of $\alpha(t)$, the two definitions of $F$ agree on $\|x\|=t$. Thus $F$ is continuous at $(x, t)$ if $(x, t) \neq(0,0)$. Since $\|F(x, t)\| \leqslant \alpha(t)$ if $\|x\| \leqslant t$ and since $\alpha(t) \rightarrow 0$ as $t \rightarrow 0, F$ is continuous at $(0,0)$. It is now easy to see that $F$ has the required properties.

REMARK. Surprisingly, the results of Section 3 imply that it may happen that $F$ cannot be chosen to be $C^{1}$ when $\mathscr{F}$ is $C^{\infty}$. We suspect that, if $\mathscr{F}$ is $C^{1}$ and $\mathscr{F}^{\prime}(k)=0$, then $F$ can be chosen to be $C^{\prime}$. The difficulty is to keep smoothness at $(s, 0)$ where $s \in M$. It can be shown with care that $F$ is $C^{\prime}$ except possibly on $M \times\{0\}$ and that $F$ is differentiable at $(s, 0)$ if $s \in M$.

We now want to return to discuss the $N_{i}$ 's.

Proposition 2. The following properties are equivalent: (a) $N_{i} \neq\{0\}$; (b) there is a $K^{i}$-invariant vector field on $M$ which does not vanish at $k$; (c) the component of $(G / K)^{K^{i}}$ containing $e K$ is positive dimensional.

Proof. The proof that (a), (b) and (c) are equivalent is essentially the same as the proof of the proposition in [6]. (Note that there is a misprint in [6]. Part (i) should read " $\operatorname{dim} N\left(G_{k}\right)=\operatorname{dim} G_{k}{ }^{\prime}$ ") Thus we merely sketch it. If $v(x)$ is a $K^{j}$ invariant vector field on $M$, then $v(k)$ is in $T_{k}(M)$ and is fixed by $K^{i}$. Thus (b) implies (a). Assume (a) holds and choose $v \in N_{i} \backslash\{0\}$. Choose a smooth vector field $\tilde{v}$ on $M$ such that $\vec{v}(k)=v$. By using the usual averaging with respect to an invariant Haar measure on $K^{i}$ (also using a $K^{i}$-invariant Riemannian metric on $M$ ), we can assume that $\tilde{v}$ is $K^{i}$-invariant. (Since $\tilde{v}(k)$ is fixed by $K^{i}$, it will not be changed by the averaging.) Thus (b) holds. Note that the above argument ensures that $\tilde{v}$ can be chosen smooth in (b). Assume that (b) holds and let $Z(t)$ be the solution of $x^{\prime}(t)=-\tilde{v}(x(t)), x(0)=k$. As in [6], we find that $Z(t)$ is a non-constant curve in $M$ fixed by $K^{i}$. Since $M$ is $G$-invariantly diffeomorphic to $G / K$ by a diffeomorphism $\phi$ which sends $k$ to $e K$, (c) holds. Conversely, if (c) holds, there must be a smooth curve $w(t)$ in $(G / K)^{K^{i}}$ such that $w(0)=e K$ and $w^{\prime}(0) \neq 0$. (Remember that each component of $(G / K)^{K^{i}}$ is a manifold.) Then $\tilde{w}(t)=\phi^{-1} w(t)$ 
is a curve in $M$ such that $\tilde{w}(0)=k, \tilde{w}^{\prime}(0) \neq 0$ and $\tilde{w}(t)$ is fixed by $K^{i}$. Hence $\tilde{w}^{\prime}(0) \in N_{i}$ and hence (a) holds.

Condition (c) shows that whether $N_{i}=\{0\}$ depends only on the three groups $G, K, K^{i}$. It is clear from (c) that it is very difficult to have $N_{i}=\{0\}$ if $G$ is abelian and positive-dimensional.

Finally to complete this section, we make some remarks on Theorem 4 (our main theorem). Firstly, if $T$ is a component of $\tilde{\pi}\left(N^{i}\right)$ such that $i$ and $T$ satisfy the conditions of the second statement of Theorem 4, then each $G$-invariant map $\tilde{F}$ near $\mathscr{F}$ has zeros $x$ near $M$ such that $G_{x}$ has $K^{i}$ as a subgroup. This shows that solutions must lie in certain parts of $\widetilde{T}$. Secondly, if $N_{1} \neq\{0\}$, our earlier comments imply that $N_{i} \neq\{0\}$ for $i>1$ and hence $\mathscr{F}$ is not $G$-stable.

\section{Some other results}

In this section, we consider briefly the cases where either $\mathscr{F}$ is $C^{1}$ or $F$ is a gradient mapping. ( $F$ is as in the introduction.) We use the notation of Section 2.

We say that $\mathscr{F}$ is $C^{1} G$-stable if firstly $\mathscr{F}$ satisfies the assumptions at the start of Section 2 and secondly, for every $C^{1} G$-invariant map $F: E \times R \rightarrow E$ such that $F(, 0)=\mathscr{F}$, the equation $F(x, t)=0$ has a solution $x$ near $M$ for each $t$ near 0 . (It would possibly be more natural to call this $C^{1}$ parametrized $G$-stable. In fact, our methods could be used to study all $G$-invariant maps $C^{1}$ close to $\mathscr{F}$.) As usual, $\Re(B)$ and $R(B)$ denote the kernel and range of $B$ respectively.

We assume that $F: E \times R \rightarrow E$ is $C^{1}$ and $\mathscr{F}=F(, 0)$ satisfies the assumptions at the start of Section 2. We use the notation there. Consider the equation $P_{k} F(k+u, t)=0$ where $u \in \mathscr{R}_{k}(M)$. We write $u=u_{1}+u_{2}$ where $u_{1} \in Y_{1} \equiv$ $\mathscr{R}\left(\left.P_{k} F_{1}^{1}(k, 0)\right|_{\Re_{k}(M)}\right)$ and $u_{2} \in Y_{2}$. Here $Y_{2}$ is a $K$-invariant complement to $Y_{1}$. Similarly, we let $\tilde{P}$ be a $K$-invariant projection of $\Re_{k}(M)$ onto $R\left(\left.P_{k} F_{1}^{l}(k, 0)\right|_{N_{k}(M)}\right)$. (It is well-known that these can be found. See, for example, [6].) By the implicit function theorem, the equation $\tilde{P} P_{k} F\left(u_{1}+u_{2}, t\right)=0$ can be solved (for $u_{1}, u_{2}, t$ small) to obtain $u_{2}=b\left(u_{1}, t\right)$. By uniqueness, we easily see that $b$ is $K$-invariant. Let $L$ be a $K$-invariant linear isomorphism between $Y_{1}$ and $R(I-\tilde{P})$. That $L$ exists is proved by the same argument as in the proof of Lemma 2 in [6]. Thus the equation $P_{k} F\left(k+u_{1}+u_{2}, t\right)=0$ near $(k, 0)$ reduces to

$$
B\left(u_{1}, t\right) \equiv L(I-\tilde{P}) P_{k} F\left(k+u_{1}+b\left(u_{1}, t\right), t\right)=0
$$

where $u_{2}=b\left(u_{1}, t\right)$. Note that $B$ is a $K$-invariant map of a neighbourhood of $(k, 0)$ in $Y_{1} \times R$ into $Y_{1}$. Let $W$ be the set of integers $i$ in $\{1, \ldots, m\}$ such that 
$K_{x}=K^{i}$ for some $x$ in $Y_{\backslash} \backslash\{0\}$. In other words, $\left\{K^{i}: i \in W\right\}$ are the set of orbit types for the action of $K$ on $Y_{1} \backslash\{0\}$. It follows as in Section 2 that, if $\tilde{r}$ is small, then $B\left(u_{1}, 0\right) \neq 0$ whenever $u_{1} \in S_{r}(k) \cap \bar{N}^{i} \cap Y_{1}$ and $N_{i}=\{0\}$. (Note that $b(0,0)=0$.) Note that $\tilde{\pi}$ was defined in Section 2 .

THEOREM 5. $\mathscr{F}$ is $C^{1} G$-stable if (I) $1 \notin W$ and $N_{1}=\{0\}$ or (II) there is an $i$ in $W$ and a component $T_{1}$ of $\tilde{\pi}\left(N^{i} \cap Y_{1}\right)$ such that (a) $N_{i}=\{0\}$, (b) $N_{K}\left(K^{i}\right) / K^{i}$ is finite and (c) $\operatorname{deg}_{Y^{i}}\left(B(, 0), p, Y^{i} \cap \tilde{r} \tilde{\pi}^{-1}\left(T_{1}\right)\right) \neq 0$ and (d) $\operatorname{dim} Y^{i}>0$ or (III) there is an $i$ in $W$ such that $N_{i}=\{0\}, \operatorname{dim} Y^{i}=0$ and $\left.B(, 0)\right|_{Y^{i}}$ is not a constant map. Here $Y^{i}=\tilde{r} S\left(Y_{1}\right)^{K^{i}}, p \in Y^{i} \cap \tilde{r} \tilde{\pi}^{-1}\left(T_{1}\right)$ and $N^{i}$ was defined in Section 2.

Proof. By combining the ideas above with those in Section 2, we see that it suffices to find solutions $u_{1}$ of $B\left(u_{1}, t\right)=0$ such that the isotropy group of $u_{1}+b\left(u_{1}, t\right)$ is in $K^{i}$ where $N_{i}=\{0\}$. Note that $u_{1}+b\left(u_{1}, t\right)$ and $u_{1}$ have the same isotropy group as is easily seen. Suppose (I) holds. Now $B(0, t)$ is fixed by $K$ since $B$ is $K$-invariant. Thus, if $1 \notin W$ (that is no point in $Y_{1} \backslash\{0\}$ is fixed by $K$ ), $B(0, t)=0$. Since 0 has isotropy group $K$ and $N_{1}=\{0\}$, it follows from our comments above that $F$ is $C^{l} G$-stable. If (II) or (III) holds, then by the arguments in the proof of Theorem 4, there is a solution $\tilde{u}_{1}$ of $B\left(u_{1}, t\right)=0$ in $B_{\tilde{r}}(k) \cap Y_{1}$ such that $K_{\tilde{u}_{1}}$ contains $K^{i}$ as a conjugate for each small $t$. Now it follows as in Section 2 that $N_{j}=\{0\}$ where $K^{j}=K_{\tilde{u}_{1}}$. Thus, by our comments above, the result follows.

REMARK. 1. It is easy to check that, if the conditions of Theorem 5 fail, then $\mathscr{F}$ is not $G$-stable. We conjecture that Theorem 5 is best possible in the sense that, if the conditions there fail, then there is a G-invariant $C^{1}$ function $F: E \times[0,1] \rightarrow E$ such that $F(, 0)=\mathscr{F}$ and $F(x, t) \neq 0$ for $t \neq 0$. We are only able to prove that $F$ can be found such that (A) $F$ is $G$-invariant, differentiable and $C^{\prime}$ for $t \neq 0,(B)$ there is a sequence $t_{i}$ approaching zero such that $F\left(x, t_{i}\right) \neq 0$ on $\sigma$ and (C) the equation $P_{k} F(k+u, t)=0$ for $u \in \mathfrak{N}_{k}(M)$ reduces to a bifurcation equation as above. Note that the proof of Theorem 5 does not really need the existence of the partial derivative $F_{2}^{1}(x, t)$.

2. It can happen that $\mathscr{F}$ is not $G$-stable but $\mathscr{F}$ is $C^{l} G$-stable. We prove this by constructing $\mathscr{F}$ not $G$-stable such that $\mathscr{F}$ satisfies the assumptions of Theorem 5. We construct an example with $G=K=S_{n}$, the symmetric group of order $n$, $k=0$ and $M=\{k\}$. (Thus the conditions on $N_{i}$ and the normalizers are trivially satisfied.) We use the representation in [21, page 35] which acts on $R^{n}$. Now, by Theorem 1, there is a continuous invariant map $\tilde{f}_{1}$ of the sphere $\tilde{S}_{r}(0)$ of radius $r$ in $R^{n}$ into itself such that $\tilde{f}_{1}$ is not $K$-invariantly homotopic to a constant and such that $\operatorname{deg}\left(\tilde{f}_{1} \mid \tilde{s}_{\text {, }(0)} K^{i}\right)=0$ for $i \in W$. [The important point is that, in this case, 
there is an $i \in W$ such that $\operatorname{dim} \tilde{N}_{i}>1$ and $\tilde{X}_{\left(K^{i}\right)} / K$ is not connected. (Here $\tilde{X}_{\left(K^{i}\right)} / K$ denotes the set of points in the quotient space $\tilde{S}_{r}(0) / K$ corresponding to the points with isotropy group conjugate to $K^{i}$.) Using Theorem 2 here, we simply choose a map corresponding to $\left\{\alpha_{j}\right\} \in A\left(R^{n},\{0\}\right)$ such that not all the $\alpha_{j}$ 's are zero but such that, for each $i \in W$, the sum of the $\alpha_{j}$ 's corresponding to $\tilde{X}_{\left(K^{\prime}\right)} / K$ is zero.] By the usual smoothing arguments, we may assume that $\tilde{f}_{1}$ is smooth. We extend $\tilde{f}_{1}$ smoothly and $K$-invariantly to $\tilde{B}_{r}(0)$ by defining $\tilde{f}_{1}(x)=$ $g(\|x\|) \tilde{f}_{1}(r x /\|x\|)$, where $g(y)=\exp \left(y^{-2}\right)$. Note that $\tilde{f}_{1}$ only vanishes at $k$. We define $\mathscr{F}: R^{n} \oplus R^{n} \rightarrow R^{n} \oplus R^{n}$ by $\mathscr{F}(x, y)=\left(x, \tilde{f}_{1}(y)\right)$. Here $K$ acts as above on each component of the direct sum. It is easy to see that $\mathscr{F}$ is smooth, $K$-invariant and only vanishes at $(0,0)$. Now the bifurcation equation above (for $t=0$ ) is easily seen to be $\tilde{f}_{1}(y)=0$. (Note that $Y_{1}=R^{n}$.) Hence our choice of $\tilde{f}_{1}$ above and Theorem 5 imply that $\mathscr{F}$ is $C^{\prime} G$-stable. On the other hand, $\mathscr{F}$ is not $G$-stable. To see this, we must show that $\mathscr{F}_{S_{r}(E)}: S_{r}(E) \rightarrow E \backslash\{0\}$ is $K$-null homotopic, where $S_{r}(E)$ denotes the sphere of radius $r$ in $E=R^{n} \oplus R^{n}$. By Theorem 8.4 and Proposition 8.3 in [21], it suffices to prove that the map $x \rightarrow r\|\mathscr{F}(x)\|^{-1} \mathscr{F}(x)$ has zero degree as a map of $S(E)^{K^{\prime}}$ into itself for each isotropy group $K^{i}$. However, by the product theorem for the degree, this degree equals the degree of $\tilde{f}_{1}$ as a map of $S\left(R^{n}\right)^{K^{\prime}}$ into itself and hence is zero by the choce of $\tilde{f}_{1}$.

3. If the construction above is examined closely, one finds that the key point is that (A) here is an isotropy group $K^{i}$ for the action of $K$ on $S\left(Y_{1}\right)$ such that $\operatorname{dim} S\left(Y_{1}\right)^{K^{i}} \geqslant 1$ and $\tilde{\pi}\left(N^{i} \cap Y_{1}\right)$ has strictly more components than $N^{i} / K$. (This will become clear from our comments below.) Necessary conditions for this to occur are that $\tilde{\pi}\left(N^{i} \cap Y_{1}\right)$ is not connected and that there is an isotropy group $K^{j}$ for the action of $K$ on $Y_{1}$ such that $K^{j} \supset K^{i}, \operatorname{dim} S\left(Y_{1}\right)^{K^{j}}=\operatorname{dim} S\left(Y_{1}\right)^{K^{i}}-1$ and $\operatorname{dim} S\left(Y_{2}\right)^{K^{j}}<\operatorname{dim} S\left(Y_{2}\right)^{K^{i}}$. Conversely, if Condition $\mathrm{A}$ above fails and if $\mathscr{F}$ satisfies the assumptions of Theorem 5 , then it can be shown that $\mathscr{F}$ is $G$-stable. The key point of the proof of this is to show that, if $\mathscr{F}$ is as above, if $H\left(=K^{i}\right)$ is an isotropy group for the action of $K$ on $S(V)$ such that some point of $S\left(Y_{1}\right)$ has isotropy group $H$, if $N_{i}=\{0\}$, if $T$ is a component of $N^{i} / K$ and if $p \in \tilde{\pi}^{-1}(T) \cap$ $S\left(Y_{1}\right)^{H}$, then

$$
\operatorname{deg}_{S_{r}(k)^{H}}\left(\tilde{F}, p, \tilde{\pi}^{-1}(T)\right)= \pm \operatorname{deg}_{S\left(Y_{1}\right)^{H}}\left(B(, 0), p, \tilde{\pi}^{-1}(T) \cap r S\left(Y_{1}\right)^{H}\right) .
$$

Here $\tilde{F}(x)=\left\|P_{k} \mathscr{F}(x)\right\|^{-1}\left\|P_{k} \mathscr{F}(x)\right\|$. This seems most easily proved by intersection theory and is an analogue of the known result that a local index is determined up to sign by the bifurcation equations (see [8], Section 3). Note that one can easily show that $\tilde{\pi}^{-1}(T) \cap S\left(Y_{1}\right)^{H}$ is non-empty.

4. A variant of Theorem 5 can be proved which gives a lower dimensional bifurcation equation if $\mathscr{R}\left(\mathscr{F}^{\mathrm{l}}(k)\right) \cap R\left(\mathscr{F}^{\mathrm{l}}(k)\right) \neq\{0\}$. We replace the equation $P_{k} F(k+u, t)=0$ by $\bar{P}_{k} F(k+u, t)=0$ where $\bar{P}_{k}$ is a $K$-invariant projection 
onto $R\left(\mathscr{F}^{\mathrm{f}}(k)\right)$. We can then argue as before to reduce to a $K$-invariant bifurcation equation $\tilde{B}\left(u_{1}, t\right)=0$ where $\tilde{B}: \mathscr{N}\left(\mathscr{F}^{1}(k)\right) \cap \mathscr{N}_{k}(M) \times R \rightarrow R\left(I-\bar{P}_{k}\right)$. By Lemma 2 in [6], there is a subspace of $R\left(I-\bar{P}_{k}\right)$ which is $K$-invariantly isomorphic to $T_{k}(M)$. Choose a $K$-invariant projection $\bar{P}$ onto this subspace such that $\bar{P} \bar{P}_{k}=0$. It is then not difficult to prove an analogue of Theorem 5 with $B$ replaced by $(I-\bar{P}) \tilde{B}$ (more strictly a linear operator times this) and $Y_{1}$ replaced by $\mathscr{N}\left(\mathscr{F}^{1}(k)\right) \cap \mathscr{R}_{k}(M)$.

We now very briefly consider the gradient case. Once again we obtain a partial result for this case. We assume that the conditions of Section 2 hold, that there is a scalar product $\langle$,$\rangle on E$ and that a map $f: E \times R \rightarrow R$ exists such that $F(, t)$ is the gradient of $f(, t)$ for each $t$ (with respect to $\langle$,$\rangle ). We discuss when F(, t)$ has a zero near $k$ for each $t$ near 0 .

Firstly, as in [8, Section 1], we may assume that $\langle$,$\rangle and f$ are $G$-invariant. Now, if $u \in \Re_{k}(M), u$ is near $k$ and $P_{k} F(u, t)=0$, then $F(u, t)=0$. To see this, we note that, by differentiating $f(x, t)$ along the orbit through $u,\langle F(u, t), z\rangle=0$ whenever $z$ is tangent to the orbit $G(u)$ at $u$. (Remember that $f$ is constant on orbits.) Since it is easily shown that $T_{u}(G(u))$ and $\Re_{k}(M)$ span $E$, it follows that $F(u, t)=0$. (A similar argument appears in the proof of Theorem 6 in [10].) Thus we see that, in the gradient case, the tangential conditions are not needed. This is a major difference from the non-gradient case. Now it is easily checked that $\left.P_{k} F(, t)\right|_{\Re_{k}(M)}$ is gradient of $\left.f(, t)\right|_{\gamma_{k}(M)}$ for each $t$. Thus our problem reduces to the case where the group acts trivially on $k$. Note that we must consider the most general problem on $\mathfrak{K}_{k}(M)$ because a $K$-invariant gradient mapping $F_{1}$ on $\mathfrak{R}_{k}(M)$ can be easily used to generate a $G$-invariant gradient map $\mathscr{F}$ near $M$ such that $P_{k} \mathscr{F}_{\mathfrak{R}_{k}(M)}=F_{1}$.

We now consider this reduced problem. Note that, by our assumptions and the results of the previous paragraph, $k$ is an isolated solution of $P_{k} F(u, 0)=0$ in $\Re_{k}(M)$. We want to use Conley's homotopy index [4]. Thus we need to assume that $F(x, 0)$ is locally Lipschitz in $x$. (This is needed to ensure that solutions of certain ordinary differential equations are unique. We suspect that it should be possible to use quasi-gradient flows as in Rabinowitz [19] to at least partially remove this assumption.) Now one easily sees that, if $1 \leqslant i \leqslant m$, then $\tilde{N}_{i}=$ $\Re_{k}(M)^{K^{i}}$ is $P_{k} F(, t)$ invariant. Moreover, $\left.P_{k} F(, t)\right|_{\tilde{N}_{i}}$ is gradient of $\left.f(, t)\right|_{\tilde{N}_{i}}$. Since $k$ is an isolated solution of $P_{k} F(, 0)=0$, it follows as in [7] that $k$ is an isolated invariant set for the flow corresponding to $P_{k} F(, 0)$ on $\tilde{N}_{i}$. Thus the homotopy index $h_{\tilde{N}_{1}}\left(\left.P_{k} F(, 0)\right|_{\tilde{N}}, k\right)$ is defined. Now the homotopy index is homotopy invariant. Hence, if $h_{\tilde{N}_{i}}\left(\left.P_{k} F(, 0)\right|_{\tilde{N}_{i}}, k\right) \neq 0$, then, for each small $t$, the equation $P_{k} F(u, t)=0$ has a solution in $\tilde{N}_{i}$ near $k$. This is similar to the proof of Theorem 2 in [7]. (Technically, the above argument is only valid if $P_{k} F(, t)$ is Lipschitz in $t$ for each $t \neq 0$. However, as in [7], this condition for $t \neq 0$ can be 
avoided by an approximation argument.) It follows from this and the results of the previous paragraph that, if there is an $i$ such that $h_{\tilde{N}_{i}}\left(P_{k} F(, 0), k\right) \neq 0$, then, for each small $t$, the equation $F(x, t)=0$ has a solution near $k$. Thus, in this case, the manifold $M$ is a stable set of zeros of $F(, 0)$ for $G$-invariant gradient mappings.

It might be objected that, since we have used invariant subspaces, we have not used the full symmetry structure. Now, for $K$-invariant gradient mappings, we could follow the procedure in [4] and define a $K$-invariant homotopy index. (We consider index pairs $(X, A)$ where $X$ and $A$ are $K$-invariant and define the $K$-homotopy index to be the $K$-invariant homotopy type of $X / A$.) However, unlike the case of Section 2, we do not gain much because a theorem of James and Segal [15] implies that, if $X / A$ is a $K$ - ENR, then $X / A$ is $K$-invariantly contractible if and only if the fixed point set $(X / A)^{K^{i}}$ is contractible for each isotropy group $K^{i}$. (The term $K-$ ENR is defined in [15].) Thus if $X / A$ is "reasonable" (and a variant of a construction of Rabinowitz [19] and a theorem of Jaworowski [16] imply that this always holds if $F(, 0)$ is $C^{1}$ ), then we get the same result as in the previous paragraph.

If $F$ is also $C^{1}$, then, by a similar argument to that in [19], the bifurcation equation $B\left(u_{1}, t\right)=0$ has a gradient structure. Moreover, the homotopy index of $\left.P_{k} F(, 0)\right|_{\tilde{N}_{i}}$ is a suspension of the homotopy index of $\left.B(, 0)\right|_{\tilde{N}_{i} \cap Y_{1}}$ (see [1]). It is unclear if any of these last homotopy indices can be non-zero while $h_{\tilde{N}_{i}}\left(\left.P_{k} F(, 0)\right|_{\tilde{N}_{i}}, k\right)=0$ for every $i$. However the neighbourhood constructed by Rothe [20] and an argument in [8, Section 1] imply that this cannot happen if (a) $F(, 0)$ is analytic or $(b) B(, 0)$ satisfies the technical condition in [20], or (c) there is local $C^{0} K$-invariant diffeomorphism $\phi$ near 0 such that $\phi(0)=k$ and $\tilde{b} \circ \phi$ is analytic (where $B(, 0)$ is the gradient of $\tilde{b}$ ) or (d) $\operatorname{dim} Y_{1} \leqslant 2$.

However, this leaves aside the basic question. Is the above homotopy index condition the best possible condition to ensure the stability of the zero $k$ of $F(, 0)$ in the $K$-invariant gradient case? This seems nontrivial and interesting even in the much simpler case where $K=G=\{e\}$ (that is, the case where there are no symmetries).

\section{Some special cases and examples}

We present a number of special cases and examples of our main theorem (Theorem 4). These also illustrate how easy the calculations of ten are in examples. The complexity in the statement of Theorem 4 is caused by a number of awkward special cases. We assume the conditions of Section 2. 
As our first special case, assume that $M$ is not discrete and $G$ acts freely on $M$, that is $G_{k}=\{e\}$. Then $k$ is not $G$-stable for $\mathscr{F}$. (This follows because, in this case, all the $N_{i}$ 's are non-trivial since $K=G_{k}=\{e\}$ and $T_{k}(M) \neq\{0\}$.) Similarly, if $G=T^{n}$ (that is an $n$-dimensional torus) and if $G_{k} \neq\{e\}$, then $k$ is not $G$-stable for $\mathscr{F}$. (This follows because $(G / K)^{K^{i}}=G / K$ if $K^{i} \subseteq K \subseteq G$ since $G$ is abelian. Proposition 2 at the end of Section 2 then implies that all the $N_{i}$ 's are non-trivial.) Note that the two results above are cases where the Golubitsky-Schaefer theory does not apply.

Next, we present a special case of Theorem 4 which covers a great many applications. Assume that $K$ is abelian or that every non-trivial irreducible subrepresentation of the action of $K$ on $N_{k}(M)$ occurs with multiplicity at least 2. (More generally, it would suffice to assume that each isotropy group $K^{i}$ for the action of $K$ on $N_{k}(M)$ with $i>1$ has the property that $\tilde{\pi}\left(N^{i}\right)$ is connected or is discrete.) Then $k$ is $G$-stable for $\mathscr{F}$ if and only if we can find an isotropy group $K^{i}$ for the action of $K$ on $N_{k}(M)$ such that

(i) $\operatorname{dim} \Re_{G}\left(K^{i}\right)=\operatorname{dim} K^{i}$ and

(ii) index $\tilde{N}_{i}\left(P_{k} \mathscr{F}_{\mid \tilde{N}_{i}}, k\right) \neq 0$.

Here $\Re_{G}\left(K^{i}\right)$ denotes the normalizer of $K^{i}$ in $G, P_{k}$ is the orthogonal projection onto $N_{k}(M)$ and

$$
\tilde{N}_{i}=\left\{x \in N_{k}(M): T_{g} x=x \text { for } g \in K^{i}\right\} .
$$

Note that $\mathscr{T}_{G}\left(K^{i}\right)$ is a manifold and that $P_{k} \mathscr{F}$ maps $\tilde{N}_{i}$ into itself and hence the index of the isolated solution $k$ is defined. (This result follows from Theorem 4, the remark immediately after the statement of Theorem 4 and a comment preceding Theorem 2 in Section 1.) The advantage of this special case is that the indices are indices on linear spaces. These spaces usually have smaller dimension than $E$. The only occasion when we have to evaluate an index on all of $E$ is when $k=0, G$ is discrete and $\{e\}$ is an isotropy group. If $G$ is discrete, (i) can be omitted. If $G$ is not discrete, one usually finds that (i) is only satisfied for a few isotropy groups $K^{i}$.

Let us consider some simple examples where $k=0$. Thus $K=G$. If 0 is the only point of $E$ fixed by all the $T_{g}$ for $g$ in $G$, then 0 is $G$-stable for $\mathscr{F}$ (since any $G$-invariant map must vanish at zero). Let us now assume that $G=S^{1}$. In this case, condition (i) can only be satisfied if we take $K^{i}=S^{1}$. (This follows by similar arguments to those in the second paragraph of this section.) Thus, in this case, 0 is $S^{1}$-stable for $\mathscr{F}$ if and only if index $E^{c}\left(\left.\mathscr{F}\right|_{E^{G}}, 0\right) \neq 0$ where, as before,

$$
E^{G}=\left\{x \in E: T_{g} x=x \text { for } g \in G\right\} .
$$

(Here $G=S^{1}$.) Thus, in this case, we have only to check a single degree to check $S^{1}$-stability. Note that $E^{G}$ may be of quite small dimension while $E$ has large dimension. If the representation of $S^{l}$ on $E$ contains many different irreducible 
subrepresentations, the structure of $S^{1}$-invariant maps of $E$ into itself may be quite complicated and the universal $S^{1}$-unfolding may contain many parameters. Thus applying the methods in [14] can be quite difficult to apply on occasions when our method is very easy to apply. As a second example, assume that $G=Z_{6}$. Then 0 is $Z_{6}$-stable for $\mathscr{F}$ if and only if index $E^{H}\left(\left.\mathscr{F}\right|_{E^{H}}, 0\right) \neq 0$ for some subgroup $H$ of $Z_{6}$. Thus $H$ can be $\{0\},\{0,3\},\{0,2,4\}$, or $Z_{6}$. (If the second or third subgroup does not occur as an isotropy group, one does not need to evaluate the corresponding index.)

Finally, note that our method in Section 3 for constructing maps which are $C^{1}$ $G$-stable but not $C^{0} G$-stable depends crucially on using representations which do not satisfy the assumption in the special case of Theorem 4 stated above.

\section{Appendix}

In this appendix, we show how Rubinsztein's methods can be used to prove Theorems 1-3. Here we assume a good deal of familiarity with [21] and, moreover, that the reader has a copy of [21] available when he reads this appendix. We follow his notation closely.

We first consider Theorem 1. The proof follows the proof of Theorem 7.2 in [21]. We point out the changes that have to be made. We first look at the proof of Lemma 4.1 there. The fixed points of $f_{1}$ are $S\left(V_{0}\right) \backslash \dot{D}_{2}$ and $\left\{y_{i}\right\}_{i=1}^{k}$. By a simple, but tedious, calculation $\left(y_{i}, 0\right)$ is a fixed point of $\phi^{\prime}(f)$ for $i=1, \ldots, k$ and these and $S(V) \backslash D_{3}$ are the only fixed points of $\phi^{\prime}(f)$. (There are several misprints in the proof of Lemma 4.1 but these are easily corrected.) Since $h(z)=2>1$ on $D_{2}$, a simple calculation shows that $\left(I-d\left(\phi^{\prime}(f)\right)_{\left(y_{i}, 0\right)}\right)$ is a direct sum of $\left(I-d\left(f_{1}\right)_{y_{i}}\right)$ and $-I_{k}$ where $k=\operatorname{dim} V_{1}$. In particular,

$$
\operatorname{det}\left(I-d\left(\phi^{\prime}(f)\right)_{\left(y_{i}, 0\right)}\right)=(-1)^{k} \operatorname{det}\left(I-d\left(f_{1}\right)_{y_{i}}\right) .
$$

A similar result holds for $d\left(\phi_{D}(f)\right)_{\left(y_{i}, 0\right)}$ since it is similar to $d\left(\phi^{\prime}(f)\right)_{\left(y_{i}, 0\right)}$. (Recall that $\phi_{D}(f)=g_{1} \circ \phi^{\prime}(f) \circ g_{1}^{-1}$.) Moreover, since $g_{1}, \phi^{\prime}(f)$ and $g_{1}^{-1}$ are $G$-invariant, they map $S\left(V_{0}\right)$ into itself. It follows that, if $\tilde{V}$ is a subspace of $V$, if $V_{0} \subseteq \tilde{V} \subseteq V$ and if $\tilde{V} \cap S(V)$ is invariant under $\phi^{\prime}(f)$ and $g_{1}$, then

$$
\operatorname{det}\left(I-d\left(\left.\phi_{D}(f)\right|_{\tilde{V} \cap S(V)}\right)_{\left(y_{i}, 0\right)}\right)=(-1)^{\operatorname{dim} \tilde{V}-\operatorname{dim} V_{0}} \operatorname{det}\left(I-d\left(f_{1}\right)_{y_{i}}\right) .
$$

We will need these results in a moment.

We now consider his Lemma 4.3. This is where the major change must be made in his proof. Firstly, in the statement of the lemma, we must change the definition 
of $\mu_{K}[f]$ to $\mu_{K}[f]=1+(\operatorname{deg}(f)-1) \Sigma(-1)^{t_{i}} \chi\left(\alpha_{i}\right)$, where $\alpha_{i}$ are the components of $(G / H)^{K}, t_{i}=\operatorname{dim} \alpha_{i}$ and the summation is over the components of $(G / H)^{K}$. With this change, the statement of Lemma 4.3 is correct. We now consider how the proof must be modified. It is easy to see that it suffices to prove the result when $K \subseteq H$. (The case where $K$ is only conjugate to a subgroup of $H$ is easily reduced to this case and the result is easy to prove if $K$ is not conjugate to a subgroup of $H$.) The part of the proof on page 17 is unchanged except that the comment on $\tilde{U}^{K}$ on the third last line should be ignored. (It is true if suitably interpreted.) Page 18 is also unchanged except that we replace the 8th line from the bottom by $s=\operatorname{dim} S(E)^{K}$. The top of page 19 down to line 16 is unchanged except that when we say $P_{i}$ is diffeomorphic to $(G / H)^{K}$ we mean componentwise. (They both consist of manifolds possibly of different dimensions.) Similarly, when we say $\tilde{U}^{K}$ is a disc bundle over $(G / H)^{K}$ we mean that it is a union of disc bundles over the components of $(G / H)^{K}$. Now $V^{K}$ is a subspace of $V$ and hence $S(V)^{K}$ is a sphere and in particular a manifold. Since $\tilde{U}$ is an open subset of $S(V)$, it follows that $\tilde{U}^{K}$ is an open subset of $S(V)^{K}$ and hence a manifold. Let $a=\operatorname{dim} \tilde{U}^{K}=\operatorname{dim} S(V)^{K}$. Recall that $\alpha_{i}$ are the components of $(G / H)^{K}$ (or equivalently of $P_{i}$ ). Now a component $T_{i}$ of $\tilde{U}^{K}$ is a disc bundle over $\alpha_{i}$. Thus the dimension of the corresponding disc must be $a-t_{i}$, where $t_{i}=\operatorname{dim} \alpha_{i}$. Similarly, the fibre of the part of the sphere bundle $\partial \tilde{U}^{K}$ over $\alpha_{i}$ will be a sphere of dimension $a-t_{i}-1$ (that is $a S^{a-t_{i}-1}$ ). Now $\partial P_{0}=\partial \tilde{U}^{K}$ is a union of bundles. Thus, by applying the argument in [21] to each component of $\partial \tilde{U}^{K}$, we find that

$$
\chi\left(\partial P_{0}\right)=\sum \chi\left(\alpha_{i}\right) \chi\left(S^{a-t_{i}-1}\right)=\sum\left(1+(-1)^{a-t_{i}-1}\right) \chi\left(\alpha_{i}\right),
$$

where the summation is over the components $\alpha_{i}$ of $(G / H)^{K}$. By repeating the argument in [21], it follows that

$$
I_{0}^{\prime}=\chi\left(P_{0}\right)=1+(-1)^{a}+\sum(-1)^{a-t_{i}-1} \chi\left(\alpha_{i}\right) .
$$

The last paragraph of page 19 is true except that (c) should be ignored.

Suppose $z \in P_{i}$. We can choose the fibres $N_{i,}$ to be locally the intersection of a subspace containing $E^{H}$ with $S(E)$ (or more strictly a conjugate of $E^{H}$ ). Thus, by the last comment in the paragraph above discussing the proof of Lemma 4.1 in [21] and by the construction of $\Phi_{U}$ in [21], we see that $\operatorname{det}\left(I-d\left(\left.h\right|_{N_{i_{2}}}\right)_{z}\right)=$ $(-1)^{y} \operatorname{det}\left(I-d\left(\left.h\right|_{S(E)^{H}}\right)_{\left(v_{i}, 0\right)}\right)$, where $y=\operatorname{dim} N_{i_{z}}+1-\operatorname{dim} E_{H}$ and $z$ is on the orbit through $v_{i}$. (Note that we really should have conjugates in here but they will not affect the determinant. The +1 occurs because $N_{i_{2}}$ is the intersection of a subspace with $S(E)$.) Note that $y$ is constant on components of $P_{i}$ but that $y$ may be different on different components. However, $\operatorname{dim} \alpha_{i}+\operatorname{dim} N_{i_{2}}=\operatorname{dim} \tilde{U}^{k}=a$ 
if $z \in \alpha_{i}$ since $\tilde{U}^{k}$ is a bundle over $\alpha_{i}$ with fibre $N_{i_{z}}$. Thus $y=\operatorname{dim} N_{i_{2}}+1-$ $\operatorname{dim} E_{H}=a-t_{i}+1-b$, where $b=\operatorname{dim} E^{H}$. Hence

$$
\begin{aligned}
\operatorname{sgn} \operatorname{det}\left(I-d\left(\left.h\right|_{N_{i_{z}}}\right)_{z}\right) & =(-1)^{a-b-t_{i}+1} \operatorname{sgn} \operatorname{det}\left(I-d\left(\left.h\right|_{S(E)^{H}}\right)_{\left(v_{i}, 0\right)}\right) \\
& =(-1)^{a-b-q-t_{i}+1} \operatorname{sgn} \operatorname{det}\left(I-d\left(\left.h\right|_{S\left(\dot{E}^{K}\right)^{K}}\right)_{v_{i}}\right),
\end{aligned}
$$

where $q=\operatorname{dim} E^{K}-\operatorname{dim} E^{H}$. For the last equality we have again used our remarks on Lemma 4.1 (with $V=E^{H}$ and $\tilde{V}=E^{K}$ ). Now, on $S(E)^{K}, h=k$ and $q=s+1-b$. Thus we eventually have that

$$
\text { sgn det }\left(I-d\left(\left.h\right|_{N_{i_{z}}}\right)_{z}\right)=(-1)^{a-s-t_{i}} \operatorname{sgn} \operatorname{det}\left(I-d k_{v_{i}}\right) \text {. }
$$

The remainder of the proof of Lemma 4.3 now follows that in [21]. The only thing we have to do is ensure that, if we are applying results for manifolds, we must apply them to each component and then take the sum. This completes the proof of Lemma 4.3. The remainder of the proof of Theorem 1 follows the proof of the corresponding result in [21].

We now prove Theorem 2. As we commented in Section 1 , the degrees are constants on $G$-homotopy classes. Thus the conditions on the degrees are necessary for $f_{1}$ and $f_{2}$ to be $G$-homotopic. Now, in [21], it is shown that any $G$-homotopy class contains a $\Phi(a)$ where $a \in A(V,\{0\})$. Thus, we have only to show that, if $a \neq b$, one of our degrees distinguishes between $\Phi(a)$ and $\Phi(b)$. We let $\left\{\left(G_{j}\right)\right\}_{j=1}^{n}$ denote the elements of $\mathscr{N}$ where, as in [21], they are ordered such that $i>j$ implies $\left(G_{i}\right) \subseteq\left(G_{j}\right)$. We define $p_{\left(G_{j}\right)}$ to be $\Sigma p_{\left(\left(G_{j}\right), \alpha\right)}$ where the summation is over the components of $\pi X_{\left(G_{j}\right)}$. (The projection $p_{\left(\left(G_{j}\right), \alpha\right)}$ are defined in [21, page 271].) Suppose we know that $p_{\left(G_{j}\right)}(a)=p_{\left(G_{j}\right)}(b)$ for $j \leqslant k-1$. We show that, if all of the degrees of $\Phi(a)$ and $\Phi(b)$ are the same, then the same equality is true for $j=k$. This will prove the result (see the proof of Theorem 8.4 in [21]). Assume $T$ is a component of $\pi X_{\left(G_{k}\right)}$ and let $\tilde{T}=\pi^{-1}(T)$. Let $x_{(H), \alpha}$ and $U_{(H), \alpha}$ be constructed as on page 28 of [21]. Let $x_{1}, U_{1}$ denote the pair corresponding to $\tilde{T}$. Choose $p \in U_{1}$. Now, by construction, $\Phi(a) x=x$ if $x \notin \cup \dot{U}_{(H), \alpha}$. Thus, by the construction of the $U_{(H), \alpha}$ 's, $x \in \tilde{T}$ and $\Phi(a) x=p$ implies that $x \in U_{1}$ or $x \in U_{\left(G_{j}\right), \alpha}$ for some $j \leqslant k-1$. Since the $U_{(H), \alpha}$ 's are disjoint, it follows that

$$
\begin{aligned}
\operatorname{deg}_{S(V)^{G_{k}}}\left(\Phi(a), p, \tilde{T} \cap S(V)^{G_{k}}\right) & =\operatorname{deg}_{S(V)^{G_{k}}}\left(\Phi(a), p, \tilde{T} \cap S(V)^{G_{k}} \cap U_{1}\right) \\
& +\sum \operatorname{deg}_{S(V)^{G_{k}}}\left(\Phi(a), p, \tilde{T} \cap S(V)^{G_{k}} \cap U_{(H), \alpha}\right),
\end{aligned}
$$

where the summation is over the $(H), \alpha$ 's corresponding to isotropy groups $G_{j}$ with $j \leqslant k-1$. However, by the construction in [21] and by our assumption that $p_{(G,)}(a)=p_{\left(G_{j}\right)}(b)$ for $j \leqslant k-1, \Phi(a)$ and $\Phi(b)$ agree on $U_{(H, \alpha)}$ if $H$ corresponds 
to $G_{j}$ for $j \leqslant k-1$. Hence

$$
\begin{aligned}
\operatorname{deg}_{S(V)} G_{k} & \left(\Phi(a), p, \tilde{T} \cap S(V)^{G_{k}} \cap U_{1}\right) \\
= & \operatorname{deg}_{S(V)^{G_{k}}}\left(\Phi(b), p, \tilde{T} \cap S(V)^{G_{k}} \cap U_{1}\right) .
\end{aligned}
$$

Suppose we replace $\Phi(a)$ by $\Phi^{\prime}(a)$ where $\Phi^{\prime}(a)=\Phi(a)$ on $U_{1}$ and $\Phi^{\prime}(a) x=x$ elsewhere. Thus the above equality is easily seen to be still true with $\Phi(a)$ replaced by $\Phi^{\prime}(a)$ (and corresponding changes for $(b)$ ) and then $\tilde{T} \cap S(V)^{G^{k}} \cap U_{1}$ replaced by $S(V)^{G_{k}}$. (This follows since we have not altered the behaviour of the functions near possible solutions. Remember that $p \in U_{1}$ and $\Phi^{\prime}(a) x=x$ outside of $U_{1}$.) We can evaluate these degrees by using $\tilde{\kappa}_{G_{k}}$. Since $\left(G / G_{k}\right)^{G_{k}}$ is finite, we find that

$$
(-1)^{p} \chi\left(\left(G / G_{k}\right)^{G_{k}}\right) a_{i}=(-1)^{p} \chi\left(\left(G / G_{k}\right)^{G_{k}}\right) b_{i},
$$

where $a_{i}\left(b_{i}\right)$ is the component of $a(b)$ corresponding to $\left(G_{k}\right), T$ and $p=$ $\operatorname{dim}\left(G / G_{k}\right)^{G_{k}}$. Because $\left(G / G_{k}\right)^{G_{k}}$ is finite and thus has non-zero Euler characteristic, it follows that $a_{i}=b_{i}$. Since we could use a similar argument for any component of $\pi X_{\left(G_{k}\right)}$, it follows that $p_{G_{k}}(a)=p_{G_{k}}(b)$ and hence the result follows.

The above method could be used to prove the injectivity of $\Phi$ in the proof of Theorem 7.2 in [21].

We now prove Theorem 3 . We first note that one can argue as in the proof of Theorem 7.2 in [21] to find that $f$ is $G$-homotopic (as a map of $Y$ into $S(V)$ ) to a map $\left.\Phi(a)\right|_{Y}$. (We simply follow the proof of the bijectivity in the proof of Theorem 7.2 in [21]. We can do this because the proof there works by changing $f$ on points of given orbit type, proceeding successively to points with "smaller" isotropy group.) As in Theorem 2, we can easily see that our degree conditions are necessary. Thus the proof reduces to showing that, if $\left.\Phi(a)\right|_{Y}$ is $G$-homotopic to $\left.\Phi(b)\right|_{Y}$, then $\left.\Phi(a)\right|_{Y}=\left.\Phi(b)\right|_{Y}$ (that is, $p_{G_{j}}(a)=p_{G_{j}}(b)$ if $G_{j}$ is an isotropy group occurring on $Y$ ). This can be proved by the same argument as in the proof of Theorem 2.

ADDED IN PROOF. The author has answered affirmatively the conjecture after Theorem 5 and answered negatively the question at the end of Section 3 with $G=\{e\}$.

\section{References}

[1] H. Amann and E. Zehnder, 'Non-trivial solutions for a class of nonresonance problems and applications to non-linear differential equations', Ann. Scuola Norm. Sup. Pisa 7 (1980), 539-603.

[2] E. Bierstone, 'General position of equivariant maps', Trans. Amer. Math. Soc. 234 (1977), $447-466$. 
[3] G. Bredon, Introduction to compact transformation groups (Academic Press, New York, 1972).

[4] C. Conley, 'Isolated invariant sets and the Morse index', CBMS regional conferences in mathematics no. 38 (Amer. Math. Soc., Providence, R.I., 1978).

[5] E. N. Dancer, 'On the existence of bifurcating solutions in the presence of symmetries', Proc. Royal Soc. Edinburgh 85A (1980), 321-336.

[6] E. N. Dancer, 'An implicit function theorem with symmetries and its applicaiton to nonlinear eigenvalue problems', Bull. Austral. Math. Soc. 21 (1980), 81-91.

[7] E. N. Dancer, 'On the existence of solutions of certain asymptotically homogeneous problems', Math. Z. 177 (1981), 33-48.

[8] E. N. Dancer, 'Symmetries, degree, homotopy indices and asymptotically homogeneous problems', Nonlinear Analysis 6 (1982), 667-686.

[9] E. N. Dancer, 'The $G$-invariant implicit function theorem in infinite dimensions', Proc. Royal Soc. Edinburgh 92A (1982), 13-30.

[10] J. Dieudonné, Treatise on analysis, Vol. IV (Academic Press, New York, 1974).

[11] A. Dold, Lectures on algebraic topology, (Springer, Berlin, 1970).

[12] M. Field, 'Transversality in G-manifolds', Trans. Amer. Math. Soc. 231 (1977), 429-450.

[13] M. Golubitsky and D. Schaefer, 'A discussion of symmetry and symmetry breaking', to appear.

[14] M. Golubitsky and D. Schaefer, 'Imperfect bifurcation in the presence of symmetry', Comm. Math. Phys. 67 (1979), 205-232.

[15] I. James and G. Segal, 'On equivariant homotopy type', Topology 17 (1978), 267-272.

[16] J. Jaworowski, 'Extension of $G$-maps and Euclidean $G$-retracts', Math. Z. 146 (1976), 143-148.

[17] K. Komiya, 'On the equivariant homotopy of Stiefel manifolds', Osaka J. Math. 17 (1980), 589-601.

[18] N. Lloyd, Degree theory (Cambridge University Press, Cambridge, 1978).

[19] P. Rabinowitz, 'A bifurcation theorem for potential operators', Functional Analysis 25 (1977), 412-424.

[20] E. Rothe, 'A relation between the type numbers of a critical point and the index of the corresponding field of gradient vectors', Math. Nachr. 4 (1951), 12-27.

[21] R. Rubinsztein, 'On the equivariant homotopy of spheres', Dissertationes Math. (Rozprawy Mat.) 134 (1976), 1-48.

Department of Mathematics

University of New England

Armidale, N.S.W. 2351

Australia 